\title{
Semiconductors : critical exponents of the unijunction transistor transition $\left(^{*}\right)$
}

\author{
J. Brini \\ Laboratoire "Physique des Composants à Semiconducteurs" $\left({ }^{* *}\right)$, E.N.S. d'Electronique et de Radioélectricité, \\ 23, avenue des Martyrs, 38031 Grenoble Cedex, France
}

(Rè'u le 5 août 1979, révisí' le 26 novembre 1979, accepté le 18 dicembre 1979)

\begin{abstract}
Résumé. - L'apparition d'une résistance négative dans le transistor unijonction est interprétée comme une transition de phase, dont on mesure les exposants critiques $\beta, \gamma^{\prime}$ et $\delta$. Loin du point critique, les exposants ont les valeurs classiques : $\beta=1 / 2, \gamma^{\prime}=1, \delta=3$. Près du point critique, $\beta$ et $\gamma^{\prime}$ diminuent fortement : $\beta=0,2$ et $\gamma^{\prime}=0,2$.

Abstract. - The negative resistance phenomenon in the unijunction transistor is interpreted as a phase transition, for which the critical exponents $\beta, \gamma^{\prime}$ and $\delta$ are measured. Far from the critical point, these exponents have classical values : $\beta=1 / 2, \gamma^{\prime}=1, \delta=3$. Close to the critical point, $\beta$ and $\gamma^{\prime}$ decrease to 0.2 and 0.2 respectively.
\end{abstract}

1. Introduction. - The unijunction transistor (double base diode) $[1,2,3]$ is a device which consists of a semiconductor bar $\left(B_{1}, B_{2}\right.$, Fig. 1) in which minority carriers are laterally injected by means of a diode (E). Because of the strong coupling between the bulk conductivity and the injector current, the device exhibits a continuous transition from a diode like current voltage characteristic to a negative resistance characteristic. This transition is governed by the interbase voltage $V_{\text {BB }}$ (Fig. 1b). Kamarinos, Viktorovitch and Berlan $[4,5,6,7]$ have mentioned an analogy in the behaviour of the parameters between this electrical transition and a liquid-gas phase transition. Figure 1 gives the correspondence of variables which characterizes this analogy.

The main difference between the two phenomena lies in the fact that one of them (the liquid-gas transition) takes place at thermodynamic equilibrium, while the other takes place far from equilibrium. Indeed, the emitter-base diode is working under strong injection conditions and the minority carriers density in the device is much higher than at equilibrium [7].

We present here an experimental study of the critical exponents [8] which characterize the unijunction transistor transition. We shall accurately define a critical point, and approach close enough to this point to establish the limits of validity of the

$\left(^{*}\right)$ La version française de cet article a été acceptée aux Comptes Rendus de l'Académie des Sciences et elle est insérée dans le $\mathrm{n}^{\circ}$ du 7 janvier 1980 .

(**) E.R.A, C.N.R.S. n" 659. proposed analogy. We shall also confirm the fact that the critical exponents are different from the classical values for transitions occurring far from thermodynamic equilibrium.

2. Critical point. - The critical point of the transition is defined by three parameters (Fig. $1 b$, point C) :

$-V_{\mathrm{BBC}}$ is the interbase voltage for which the $I_{\mathrm{E}}\left(V_{\mathrm{E}}\right)$ characteristic exhibits a vertical region (the dynamical resistance $\partial V_{\mathrm{E}} / \partial I_{\mathrm{E}}$ is rigorously zero). This voltage has been determined with a relative accuracy of $5 \times 10^{-4}$. This accuracy depends on the thermal stability of the thermostat in which the device is immersed. (In our experiment, $T=40^{\circ} \mathrm{C} \pm 0.01$.)

$-V_{\mathrm{EC}}$ is the emitter voltage corresponding to the vertical region of the curve. This voltage is determined with an accuracy of $5 \times 10^{-4}$.

$-I_{\mathrm{EC}}$ is the emitter current at the critical point. As the zero dynamical resistance part of the curve is wide, the observation alone of the critical characteristic is not sufficient to determine $I_{\mathrm{EC}}$. To obtain an accurate value of $I_{\mathrm{EC}}$, we have used three methods, based on different physical principles.

The first is derived from the method of determining the critical volume in Van der Waals isotherms plot. Let $I_{\mathrm{p}}$ and $I_{\mathrm{v}}$ be the values of the current, corresponding to the extrema of potential (Fig. 2). We define then the critical current by :

$$
I_{\mathrm{EC}_{1}}=\operatorname{Lim}_{V_{\mathrm{BB}} \rightarrow V_{\mathrm{BBC}}} \frac{I_{\mathrm{p}}+I_{\mathrm{v}}}{2}
$$

(dotted curve). 


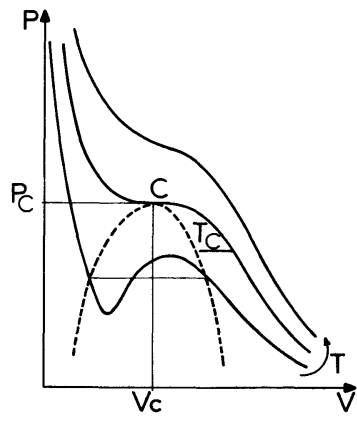

a) Liquid-gas transition.

Pressure

Molecular volume

Temperature

Reduced temperature $\quad t=\frac{T-T_{\mathrm{c}}}{T_{\mathrm{c}}}$

$P-P_{\mathrm{c}} \sim\left|\rho_{\mathrm{L}}-\rho_{\mathrm{G}}\right|^{\delta}$

$\rho_{\mathrm{L}}-\rho_{\mathrm{G}} \sim(-t)^{\beta}$

$K_{T} \sim(-t)^{-\gamma^{\prime}}$

$\rho_{\mathrm{L}}:$ Liquid density

$\rho_{\mathrm{G}}$ : Gas density

$K_{T}$ : Isothermal compressibility

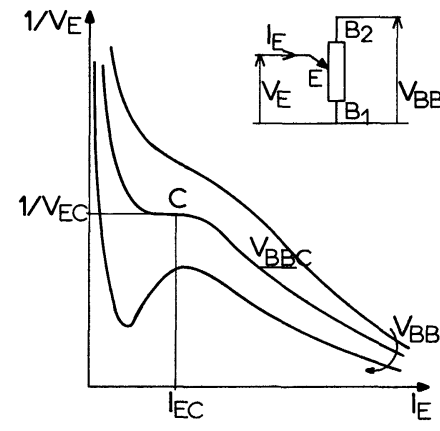

h) Unijunction transition

$\frac{1}{V_{\mathrm{E}}}$
$I_{\mathrm{E}}$
$\frac{1}{V_{\mathrm{BB}}}$

$V_{\mathrm{E}}$ : emitter voltage

: emitter current

$V_{\mathrm{BB}}:$ interbase voltage

$v=\frac{V_{\mathrm{BB}}-V_{\mathrm{BBC}}}{V_{\mathrm{BBC}}}:$ reduced interbase voltage

$\left|V_{\mathrm{E}}-V_{\mathrm{EC}}\right| \sim\left|I_{\mathrm{E}}-I_{\mathrm{EC}}\right|^{\delta}$

$\left|I_{\mathrm{b}}-I_{\mathrm{a}}\right| \sim v^{\beta}$

$\left.\frac{\partial I_{\mathrm{E}}}{\partial V_{\mathrm{E}}}\right|_{I_{\mathrm{b}}} \sim v^{\gamma^{\prime}}$

Fig. 1. - Corresponding parameters between a liquid-gas transition and the unijunction transition.

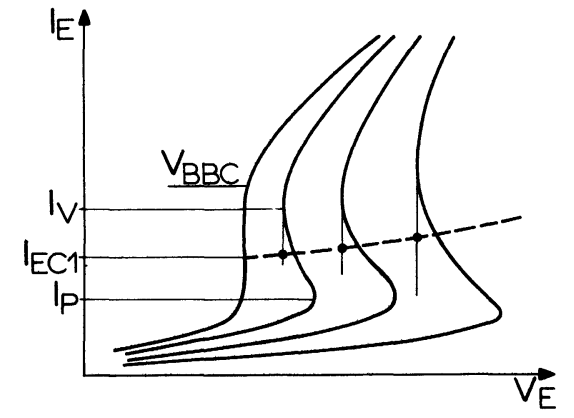

Fig. 2. - Definition of the critical current $I_{\mathrm{EC}_{1}}$.

A second method consists in plotting the emitter current fluctuations along the critical characteristic. One observes a very sharp maximum at low frequencies for a well defined value $I_{\mathrm{EC}_{2}}$ of the current $\left({ }^{1}\right)$ (Fig. 3).

A third method uses the critical exponent $\delta$, which characterizes the shape of the critical characteristic :

$$
\left|V_{\mathrm{E}}-V_{\mathrm{EC}}\right|=\left|I_{\mathrm{E}}-I_{\mathrm{EC}}\right|^{\delta} .
$$

We calculate $\delta$ for several values of $I_{\mathrm{EC}}$ on both sides of the critical point. There exists a well defined value

( $\left.{ }^{1}\right)$ We have measured the fluctuation spectra in the neighbourhood of the critical point. The results and their interpretation will be published later. In particular a correlation exponent, which would characterize the life-time of a fluctuation in the device, will be defined.

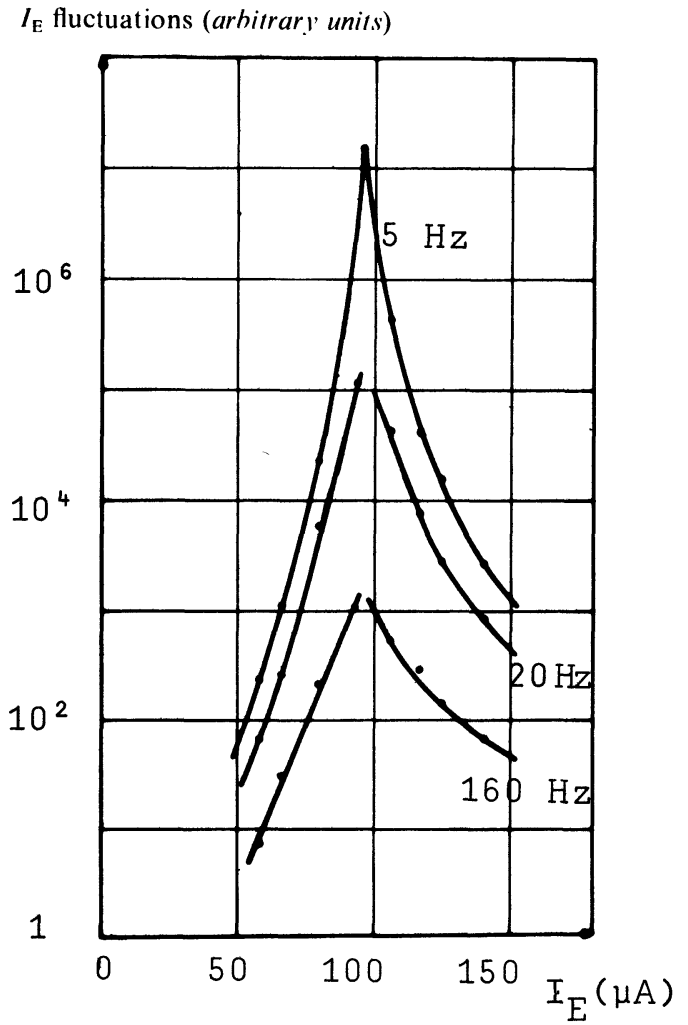

Fig. 3. - Current fluctuations in the neighbourhood of the critical point.

of the current, $I_{\mathrm{EC}}=I_{\mathrm{EC}_{3}}$, for which $\delta \# 3$ for various values of $V_{\mathrm{E}}$ lower or greater than $V_{\mathrm{EC}}$. We consider then that $I_{\mathrm{EC}_{3}}$ is the critical current. 
It is noteworthy that for a given temperature, the three values $I_{\mathrm{EC}_{1}}, I_{\mathrm{EC}_{2}}$ and $I_{\mathrm{EC}_{3}}$ are identical, with an accuracy of better than $1 \%$. The critical point is therefore well defined and allows us to compute the critical exponents.

3. Critical exponents. - In addition to the exponent $\delta$, which is defined above, our measurements concern the two exponents $\beta$ and $\gamma^{\prime}$.

- For a liquid-gas transition, the $\beta$ exponent describes the shape of the coexistence curve in the neighbourhood of the critical point [8] :

$$
\rho_{\mathrm{L}}-\rho_{\mathrm{G}}=(-t)^{\beta}
$$

with $\rho_{\mathrm{L}}$ : liquid density,

$\rho_{\mathrm{G}}:$ gas density,

$t$ : reduced temperature.

In our case we do not observe any straight line part on the characteristic as is the case in Van der Waals isotherms. If we bias the unijunction transistor without any external load, the $I_{\mathrm{E}}\left(V_{\mathrm{E}}\right)$ characteristic exhibits an hysteresis cycle abcd (Fig. 4).

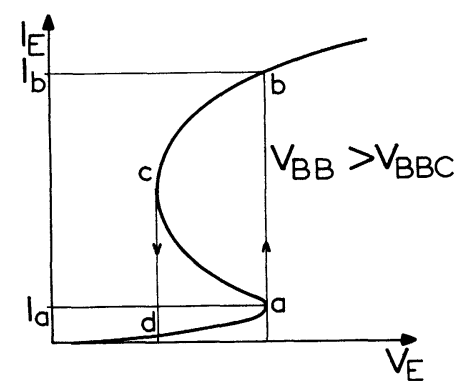

Fig. 4. - Hysteresis cycle for a negative resistance characteristic.

We have chosen to define the $\beta$ exponent as characterizing the amplitude variation of the jump ab, when approaching the critical point $\left({ }^{2}\right)$ :

$$
I_{\mathrm{b}}-I_{\mathrm{a}} \sim(v)^{\beta} \quad \text { with } \quad v=\frac{V_{\mathrm{BB}}-V_{\mathrm{BBC}}}{V_{\mathrm{BBC}}} .
$$

- The $\gamma^{\prime}$ exponent describes the evolution of the conductance at the b point (Fig. 4) (analogous of the isothermal compressibility) when one approaches the critical point :

$$
\frac{\partial I_{\mathrm{E}}}{\partial V_{\mathrm{E}}} \sim(v)^{\gamma^{\prime}}
$$

4. Results. - The measurements have been performed on bar type silicon transistors $(2 \mathrm{~N}$ 1671) [9].

- The experimental value of $\delta$ is :

$$
\delta=3.1 \pm 0.1
$$

$\left({ }^{2}\right)$ Rigorously, there are two $\beta$ exponents, depending on whether we choose the ab jump or the od jump. In our accuracy domain, we always have $\beta_{1}=\beta_{2}$. this means that the critical characteristic is a third order curve near the critical point. This result is in agreement with the mean field theories of phase transitions.

- For the $\beta$ and $\gamma^{\prime}$ exponents we can distinguish two regions, according to the value of $v$ :

a) The first is defined by $v>10^{-2}$, i.e. far from the critical point. Under these conditions, we obtain :

$$
\begin{aligned}
\beta & =0.45 \pm 0.05 \\
\gamma^{\prime} & =1.05 \pm 0.05
\end{aligned}
$$

Here also the numerical values are very close to those $\left(\beta=1 / 2, \gamma^{\prime}=1\right)$ of the mean field theories.
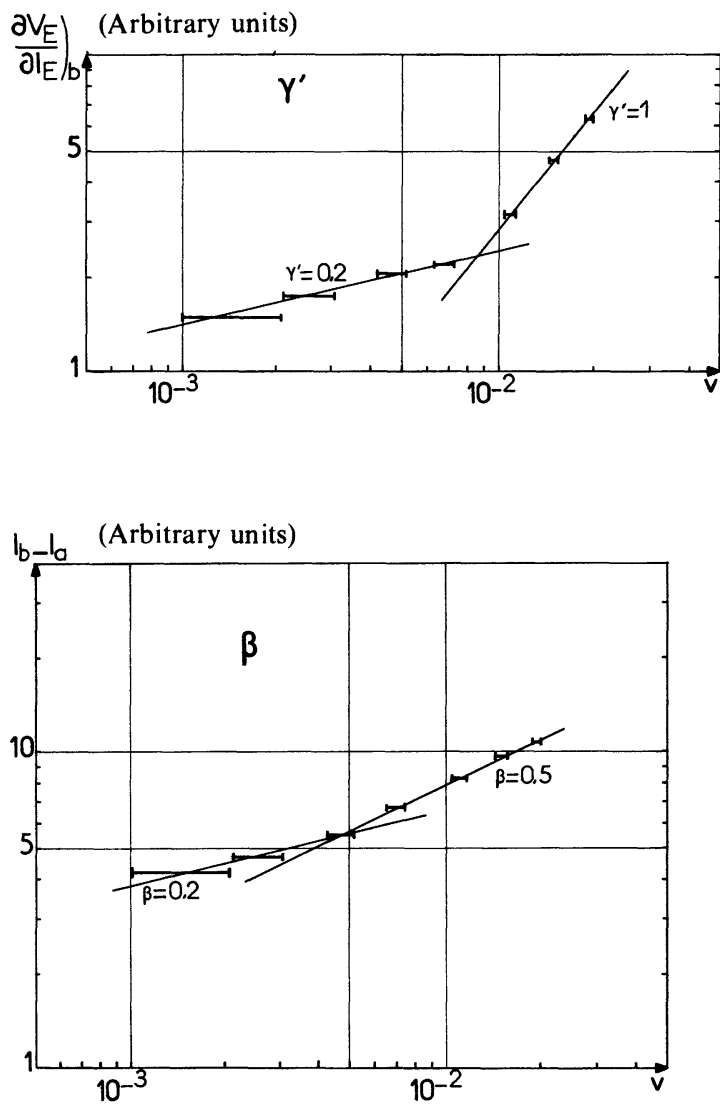

Fig. 5. - Critical exponents $\beta$ and $\gamma^{\prime}$.

b) When one approaches the critical point $\left(v<10^{-2}\right)$ one observes new values for the slope of the curves $\partial V_{\mathrm{E}} / \partial I_{\mathrm{E}}(V)$ and $\left(I_{\mathrm{b}}-I_{\mathrm{a}}\right)(v)$.

The corresponding new values of the exponents are :

$$
\begin{aligned}
& \beta=0.2 \pm 0.1 \\
& \gamma^{\prime}=0.2 \pm 0.1
\end{aligned}
$$

This new slope remains constant for any choice of $V_{\text {BBC }}$ within its confidence interval. Hence it cannot be attributed to errors due to temperature fluctuations, but must be related to the transition itself. 
5. Conclusion. - So long as one remains far enough from the critical point $\left(v>10^{-2}\right)$ the analogy between the investigated transition and a liquid-gas transition is widely corroborated by the calculation of the critical exponents. The measured values are the same as the mean field theories values.

This accordance disappears when one approaches close to the critical point. The exponents then have non-classical values. Nevertheless the analogy with other instabilities far from equilibrium, such as electroconvection [11] or the Bénard instability [10] must be considered to be only qualitative. Indeed, the value of $v\left(10^{-2}\right)$ for which the non-classical values appear in our case, is much greater than the value $\left(10^{-6}\right)$ which has been estimated for the Benard instability $[10,12]$.

\section{References}

[1] Sze, S. M., Physics of Semiconductor Devices (Wiley) 1969 , p. 310 .

[2] Scharfetter, D. L., Jordan, A. G., IRE Trans. Electron. Devices 9 (1962) 461

[3] Clark, L., Electronics, juin 1965, p. 93.

[4] Kamarinos, G., Etude des proprićtés ćlectriques d'un semiconducteur soumis d̀ un désćquilibre thermodynamique isotherme, Thèse d'Etat (juin 1974).

[5] Berlan, D., Viktorovitch, P., Kamarinos, G., " Noise measurements near an electrical transition : critical working conditions of the unijunction ", Physica 92B +C (1977) 85-88.

[6] BERLAN, D., Etude des fluctuations au voisinage d'une transition clectrique : bruit du transistor unijonction, Thèse de Docteur-Ingénieur (juillet 1977).
[7] Berlan, D., Vik torovitch, P., Kamarinos, G., " Noise in a semiconductor device working under critical conditions ", Proceedings of the Fifth Intern. Conf. on Noise (Springer-Verlag, Berlin Heidelberg) mars 1978.

[8] Stanley, H. E., Introduction to phase transitions and critical phenomena (Oxford, Clarendon Press) 1971.

[9] EimBindeR, J., "The unijunction transistor », Electronics, juin 1965, p. 87.

[10] Berge, P., Congrès de la S.F.P., Dijon 30/6-4/7/1975, " Aspects expérimentaux de l'instabilité de Rayleigh-Bénard».

[11] LACROIX, J. C., Instabilités hydrodynamiques et électro-convection lors d'injection d'ions dans les liquides isolants isotropes, Thèse d'Etat (avril 1976).

[12] Martin, P. C., Congrès de la S.F.P., Dijon 30/6-4/7/1975, "Instabilities, oscillations and chaos". 